\title{
Hypothyroidism Prolongs Hospitalization Following Surgery
}

\author{
Raquel Villavicencio', Cary N. Mariash ${ }^{1,2^{*}}$ \\ ${ }^{1}$ Department of Medicine, Division of Endocrinology, Indiana University School of Medicine, Indianapolis, IN, USA \\ ${ }^{2}$ Enterprise Clinical Research Operations, Indiana University Health, Indianapolis, IN, USA \\ Email: ${ }^{\star}$ cmariash@iu.edu
}

How to cite this paper: Villavicencio, R. and Mariash, C.N. (2019) Hypothyroidism Prolongs Hospitalization Following Surgery. International Journal of Clinical Medicine, 10, 639-650.

https://doi.org/10.4236/ijcm.2019.1012053

Received: November 19, 2019

Accepted: December 3, 2019

Published: December 6, 2019

Copyright () 2019 by author(s) and Scientific Research Publishing Inc. This work is licensed under the Creative Commons Attribution International License (CC BY 4.0).

http://creativecommons.org/licenses/by/4.0/

(c) (i) Open Access

\begin{abstract}
Objective: Each year 45 million surgical procedures are performed in the United States, and a significant number of these are performed on hypothyroid patients. Little guidance is available to determine the postoperative risk to these subjects. We hypothesized that new surgical techniques and modern anesthesia would lead to no differences in outcome between hypothyroid patients and euthyroid controls. Methods: We queried surgical databases in our health system for patients who underwent an operative procedure between January 1, 2010 and December 31, 2015 with a TSH $>10 \mathrm{mcU} / \mathrm{mL}$ or a FT4 $<$ $0.6 \mathrm{ng} / \mathrm{dL}$. Identified patients were matched to euthyroid controls selected for age, sex, surgical procedure, and search interval. Predicted length of hospital stay (LOS) was determined using the American College of Surgeons National Surgical Quality Improvement Program surgical risk calculator. Results: We identified 29 hypothyroid patients. The LOS was significantly longer for the hypothyroid patients compared to the predicted LOS (14.4 vs 6.7 days, $\mathrm{P}<$ 0.001). The LOS in the matched controls was not significantly different than their predicted LOS (9.6 vs 7.1 days, $\mathrm{P}=0.11$ ). Other complications were not different between the hypothyroid and control patients. Conclusions: In contrast to our initial hypothesis, hypothyroidism is associated with a 2 -fold longer LOS following surgery. Hypothyroidism continues to place patients at increased surgical risk.
\end{abstract}

\section{Keywords}

Hypothyroidism, Surgical Complications, Length of Stay, Preoperative Evaluation, Outcomes

\section{Introduction}

Many institutions have made the investment to provide preoperative evaluation 
for patients not requiring emergent surgery. Despite the extra care, evidence that this activity improves outcomes is controversial. Some evidence indicates that preoperative evaluation is beneficial [1] [2] [3], but at least one study suggests that these evaluations may prolong hospital stay and increase mortality [1]. We are unaware of any study that suggests a benefit to screening for hypothyroidism in the preoperative evaluation.

One of the most widely used measures to predict hospital stay length and mortality is the Elixhauser Comorbidity Index [4]. This study found that hypothyroidism was associated with a 7\% increase in hospital stay and, surprisingly, a $30 \%$ decrease in in-hospital mortality. The decrease in hospital mortality is in contrast to other studies that document increased hospital mortality with hypothyroidism [5] [6] [7]. Given the effects the thyroid has on the cardiovascular, respiratory, and gastrointestinal systems, it is intuitive that hypothyroidism might contribute to greater perioperative morbidity, as suggested by a recent review [8]. However, if greater morbidity occurs, the extent of its clinical significance has not been clearly demonstrated. This is especially important since cancelling a scheduled surgery until a patient is euthyroid may impose significant inconvenience to the patient and cost to the health-care system, as it usually means a delay of surgery for a minimum of six to eight weeks. These competing issues often make it difficult to accurately weigh the risks versus benefits of either proceeding with or cancelling surgery, posing a significant clinical dilemma to the physician performing the preoperative examination.

Investigations aimed at addressing the dilemma of whether to operate or delay the operation have previously shown conflicting results. A retrospective study conducted in 1983 that compared 59 hypothyroid patients to 59 matched controls showed no difference between controls and patients with mild to moderate hypothyroidism ( $\mathrm{TSH}>15 \mu \mathrm{U} / \mathrm{mL}$ or $\mathrm{T} 4<4 \mu \mathrm{g} / \mathrm{dL}$ ) to warrant a delay of surgery [9]. A second study conducted the following year, comparing 40 hypothyroid patients (T4 $1.9 \pm 1.0 \mu \mathrm{g} / \mathrm{dL}$ ) to 80 controls, showed an increased risk of intraoperative hypotension; heart failure in patients undergoing cardiac surgery; GI and neuropsychiatric complications; and an inability to mount a fever in the setting of infection [10]. Certainly, the most feared and potentially serious complication of performing surgery on a hypothyroid patient is the risk of precipitating myxedema coma, which, although rare, is an extreme form of hypothyroidism that can quickly escalate to death [11]. There has been at least one case report of a similar circumstance in which a 78-year-old hypothyroid man with a preoperative TSH of 25 one week prior to surgery underwent a cardiac operation that resulted in myxedema coma and life-threatening cardiac depression; on postoperative day 3, thyroid function tests showed a TSH of 13 [12].

Given the scarcity and inconsistency of data we felt further scrutiny of this topic was needed. Furthermore, because of the advances in anesthesia and surgical approaches over the last three decades, we hypothesized that length of stay would not be significantly affected in patients with mild hypothyroidism. Thus, 
we performed an up-to-date retrospective, observational study to better understand modern-day perioperative risks of hypothyroid patients, comparing the primary outcomes of length of stay, mortality, and ischemic cardiac events. Secondary outcomes included the presence or absence of fever in the setting of post-operative infection, hypothermia, hypotension, prolonged anesthetic recovery time/time to extubation, ileus, altered mentation/delirium, hyponatremia, and bradycardia.

\section{Materials and Methods}

\subsection{Study Design and Case Selection}

This study was approved by the Indiana University Institutional Review Board with the specific aim of comparing length of hospital stay between hypothyroid patients and controls. We searched Indiana University Health's electronic medical records (EMR) for patients who had undergone a surgical procedure and had a TSH above the upper limit of normal or a free T4 of $<0.6 \mathrm{ng} / \mathrm{dL}$ between January 1, 2010 and December 31, 2015. We obtained 788 charts to review. To be included in the study, patients had to be 18 years of age or older with either an elevated TSH or a low FT4 within four weeks of a major surgical procedure at one of our campus sites. If the FT4 was $<0.6$ the subject also had to have an elevated TSH. TSH values in our study cohort ranged from 7.18 to $84.736 \mathrm{mcU} / \mathrm{mL}$ with an average of $29.19 \mathrm{mcU} / \mathrm{mL}$ (standard deviation $20.54 \mathrm{mcU} / \mathrm{mL}$ ). Of the 788 screened, 29 cases satisfied our study criteria after excluding those due to patient duplication, ineligible procedures, thyroid function tests more than four weeks from surgery, and incorrect or absent thyroid function tests. Power analysis based on a predicted mean hospital stay of $7 \pm 3$ days and a $50 \%$ increase in predicted hospital stay when hypothyroid required 24 subjects at $80 \%$ power (12 subjects in each group). We also obtained a set of local euthyroid control subjects by searching the EMR for patients who had undergone similar surgical procedures during the same time interval and matched for age \pm 5 years and sex. This yielded approximately 15 potential controls for each hypothyroid subject. From the list of potential controls we randomly chose a control and reviewed that control's chart to assure they met inclusion and exclusion criteria criteria and did not differ in other confounding variables. Only that control was used for subsequent analysis. Table 1 presents the characteristics of the subjects and Table 2 gives the surgical procedures.

Two separate control analyses were conducted. In one analysis we used our matched surgical controls. The average age in both hypothyroid subjects and controls was 61.1 years. In the other analysis, each hypothyroid patient's own personal health information was entered into the American College of Surgeons National Surgical Quality Improvement Program (ACS NSQIP) surgical risk calculator [13] and the predicted length of stay from the calculator was compared against the actual length of stay. This calculator has been validated in multiple studies [14] [15] [16]. Its data is based on over 1.4 million patients cared for 
Table 1. Characteristics of the cohort of patients $(\mathrm{N}=29)$ and controls $(\mathrm{N}=29)$. Statistical comparison between patients and controls by sex and age was performed by student's t-test and for ASA class by chi-square analysis. $\mathrm{P}$ values $>0.05$ are considered non-significant (NS). NA = not applicable.

\begin{tabular}{cccc}
\hline Parameter & Patients & Controls & P value \\
Sex & & & \\
Male & 15 & 15 & NS \\
Female & 14 & 14 & NS \\
Mean Age \pm SD (years) & $61.1 \pm 12.7$ & $61.1 \pm 11.7$ & NS \\
ASA Class & & & \\
Class 2 & 2 & 3 & NS \\
Class 3 & 17 & 19 & NS \\
Class 4 & 10 & 7 & NS \\
Mean TSH \pm SD (0.4 - 4.2 mcU/mL) & $29.19 \pm 20.54$ & NA & \\
Free T4 & & NA & \\
0.2 & 2 & & \\
0.3 & 1 & & \\
0.4 & 2 & & \\
0.5 & 5 & & \\
\hline
\end{tabular}

Table 2. Types of surgical procedures performed on hypothyroid patients. The number of patients treated by surgical procedure is listed. There were an equal number of control patients for each surgical procedure listed.

\begin{tabular}{cc}
\hline Procedure Type & Number of Patients (\%) \\
\hline Laporatomy and/or Bowel Resection & $6(21)$ \\
Pharygeolaryngectomy, Esophagoectomy/Plasty & $5(17)$ \\
Wound or Bone Debridement & $5(17)$ \\
Open Reduction/Internal Fixation Fracture & $3(10)$ \\
Thoracic Corpectomy or Laminectomy & $2(7)$ \\
Hip Arthorplasty & $2(7)$ \\
LVAD Implantation and Sternotomy & $1(3)$ \\
Cystectomy with Pouch Creation & $1(3)$ \\
Nissen Fundoplication & $1(3)$ \\
Femoral Artery Ligation & $1(3)$ \\
Drainage of Subdural Hematoma & $1(3)$ \\
Hernia Repair & $1(3)$ \\
\hline
\end{tabular}

in 393 ACS NSQIP hospitals and encompasses 1557 unique CPT codes. Regression models were used to predict outcomes based on twenty-one preoperative risk factors achieving a Brier score of 0.011 for mortality and 0.069 for morbidity.

\subsection{Analysis of Data}

Every chart was reviewed by the authors to ensure data validity. Data points 
were collected on all cases and controls, including demographics (age, gender, surgery location); surgical variables (surgical procedure, date, urgency, wound class, duration of surgery, time to extubation, and estimated blood loss); patient variables (ASA Class, functional status, creatinine, dialysis, obstructive sleep apnea, chronic obstructive pulmonary disease, diabetes mellitus, congestive heart failure, coronary artery disease, previous cardiac event, hypertension, disseminated cancer, supplemental oxygen, chronic steroids, beta-blockers, calcium channel blockers, dyspnea, height, weight, smoking, sepsis, ascites, ventilator, and revised risk criteria); thyroid variables (TSH, free T4, thyroid hormone replacement, clinical assessment); and all primary and secondary outcomes. Length of stay was determined by the hospital day number at discharge regardless of the hour the patient was released. For example, if the patient was discharged on hospital day three in the morning, a length of stay of three days was ascribed. Hypothermia was defined as temperature $<35^{\circ} \mathrm{C}\left(<95^{\circ} \mathrm{F}\right)$; bradycardia as a heart rate $<60 \mathrm{bpm}$; hypotension as systolic blood pressure $<90 \mathrm{mmHg}$, diastolic blood pressure $<60 \mathrm{mmHg}$, or mean arterial pressure $<70 \mathrm{mmHg}$ intraoperatively to 48 hours post-op.

\subsection{Statistics}

Statistical evaluation was made using the computer program R [17]. Specific modules and functions in that program used are given in quotes. Data were analyzed both parametrically with a t-test ("t.test" function) and confirmed non-parametrically with the Mann-Whitney test ("wilcox.test" function). The $\mathrm{t}$-test used the Welch method which does not require the variances between groups to be equal. Where appropriate, paired t-tests were performed but results were no different from unpaired t-tests. Power calculations were made using the "power.t.test" function. Graphics were generated from the R program (using the "boxplot" function and the add-in module "ggplot2"). Linear regression analysis was also performed when appropriate (with the "lm" function). Categorical data were compared by chi-square analysis with Yates correction where applicable.

\section{Results}

\subsection{Anesthetic Considerations}

All of the case subjects' surgeries were performed under general anesthesia. Of the controls, two were done with MAC (monitored anesthesia care) and one under deep sedation. The remainder of the controls was performed under general anesthesia. The anesthetic agents most commonly used in the hypothyroid group were propofol (twenty-five of twenty-nine), fentanyl (twenty of twenty-nine), and midazolam (nineteen of twenty-nine). Etomidate and ketamine were used in a minority of patients (four and three patients, respectively). The most frequent neuromuscular blocker utilized in the hypothyroid group was rocuronium (nineteen of twenty-nine). Other agents used were succinylcholine, cisatracurium, pancuronium, and vecuronium. Similar rates of use were observed 
in the control group.

\subsection{Primary Outcomes}

We initially compared the predicted length of stay from the NSQIP risk calculator to the actual length of stay from the twenty-nine patients. We found that the hypothyroid patients had a statistically significant longer length of stay than predicted, which at 14.4 days was more than twice that of the predicted value of 6.9 days (Figure 1(a)). In order to be sure that this average length of stay was not skewed by a few patients that may have had a prolonged stay, we also looked at the number of patients whose length of stay was longer than that predicted. We found that twenty-one of the twenty-nine subjects had a statistically significantly $(\mathrm{P}<0.001)$ longer than predicted length of stay (Figure $1(\mathrm{~b})$ ).

Because the much longer length of stay surprised us, we wanted to make sure this was not due to a system wide difference in patient management in our hospitals compared to others. Therefore, we obtained a matched set of controls from our electronic medical record of patients who were not hypothyroid with the same surgical procedure and similar comorbidities. We then compared their length of stay with that calculated from the NSQIP risk calculator. These control patients had an actual length of stay of 9.6 days that was not significantly different from the predicted 7.1 days (Figure 1(a)). Moreover, the average length of
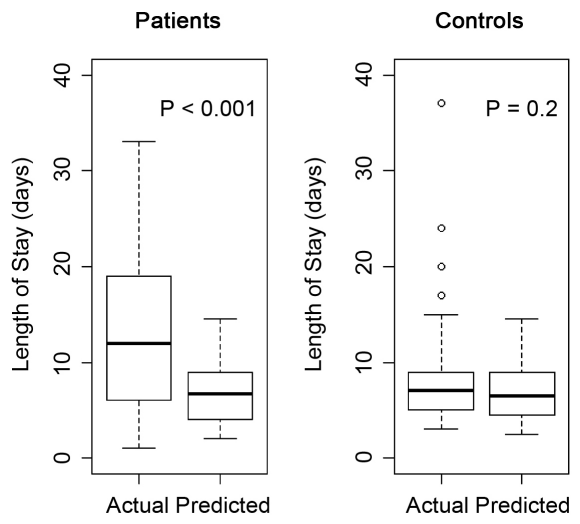

(a)

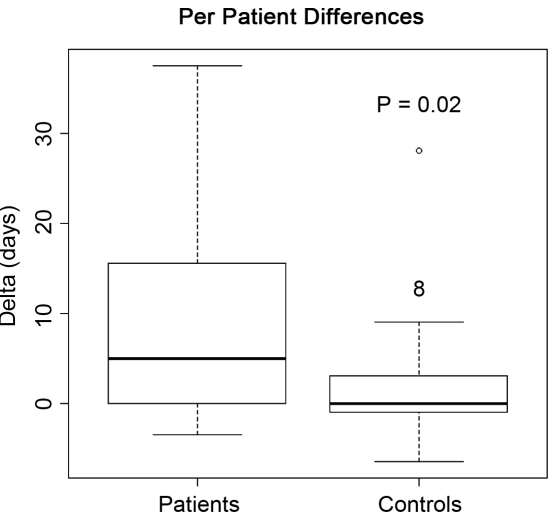

(b)

Figure 1. (a): Predicted and actual length of stay. Box plots of the actual length of stay and the NSQIP predicted length of stay. Each box shows the median and interquartile range for the listed group. The whiskers show the range. The dots indicate values that are more than 1.5 times greater than the interquartile range. The mean length of stay in the Patients group is 14.4 and 6.7 days for the Actual and Predicted, respectively. The mean length of stay in the Controls group is 9.1 and 7.1 days for the Actual and Predicted, respectively. The $\mathrm{P}$ values shown were determined by the 2 -samplettest and were confirmed by the Wilcoxan rank sum test. (b): Differences between actual and predicted length of Stay. The box plots show the median and interquartile ranges of the actual differences between each patient's length of stay and that predicted by the NSQIP calculator. The whiskers show the range of values and the dots are plotted for those values when they exceed 1.5 times the interquartile range. The mean difference is 7.8 days for the patients and 2.1 days for the Controls. The $\mathrm{P}$ value was determined by the 2 -sample $t$ test and confirmed by the Wilcoxan rank sum test. 
stay of the controls was significantly less than that of the hypothyroid patients $(\mathrm{P}=0.05)$. This difference is readily appreciated in Figure $1(\mathrm{~b})$ showing that the mean length of stay was 7 days longer than predicted in the hypothyroid patients and only 2 days longer than predicted in the controls. This difference was also statistically significant $(\mathrm{P}=0.02)$.

Since the hypothyroid patients had a significantly longer length of stay compared with the predicted length of stay, we also looked at the relationship between the length of stay and the serum TSH. We found no significant relationship between the actual length of stay or the increase in the length of stay, and the TSH or the logarithm of TSH.

We also looked at the other primary outcomes. One patient in the hypothyroid group died compared to none in the control group. Two patients in the hypothyroid group experienced atrial fibrillation. One patient in each group had pulseless electrical activity arrest. The number of these complications was too small to determine if they were different between the groups.

\subsection{Secondary Outcomes}

While five of the patients had postoperative hypothermia compared to none of the controls, the P-value for this difference was 0.06 . Even though there was no significant difference found between either group in the other outcomes (Figure 2 ), in the hypothyroid group there was a trend toward higher incidence of ileus,

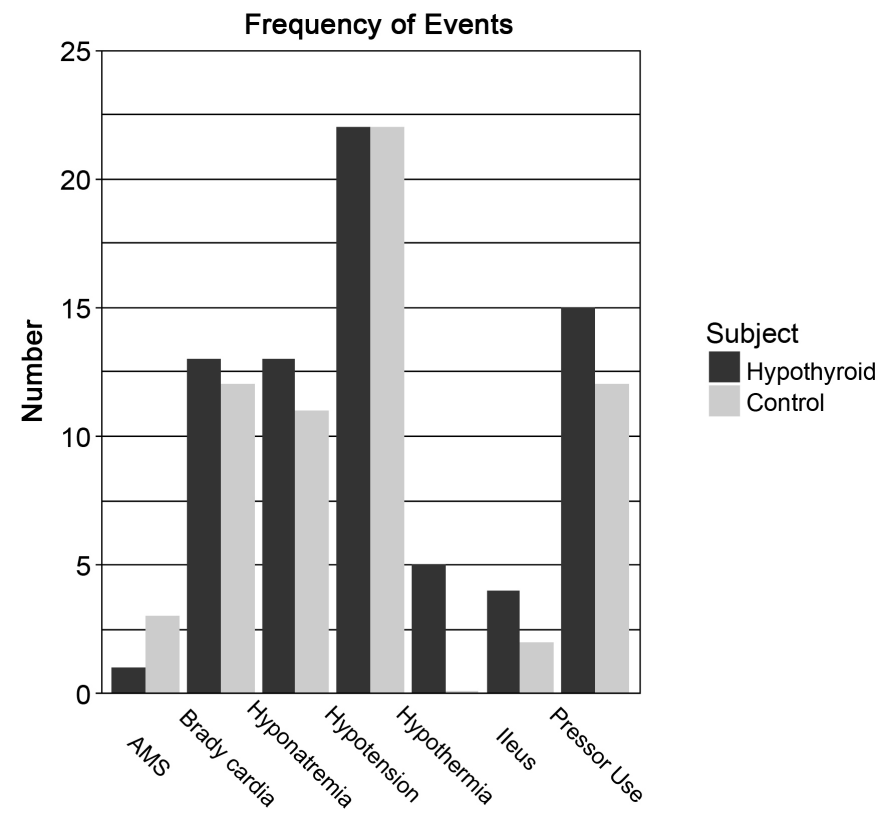

Figure 2. Secondary outcomes. Secondary outcomes are plotted against the number of occurrences of each event. There were 29 subjects in each group. The 4 patients with hypothermia were significantly different from the 0 Controls $(\mathrm{P}=0.03)$ by chi-square test, but there were no significant differences within the other events. There were 0 control subjects in both the Hypothermia and Reintubation events. AMS = altered mental status. 
use of vasopressors, and need for reintubation despite approximately equal rates of chronic obstructive pulmonary disease amongst both groups (38\% compared to $41 \%$ ) and a lower incidence of obstructive sleep apena amongst the hypothyroid group (7\% compared to $14 \%)$. We also found that four hypothyroid patients failed to mount a fever when infected. Additionally, the magnitude of hypotension was slightly more pronounced in the hypothyroid group with an average low mean arterial pressure of $51 \mathrm{mmHg}$ vs $56 \mathrm{mmHg}$ in the controls. Approximately equivalent rates of bradycardia were observed ( $45 \%$ in the hypothyroid group vs $41 \%$ in the controls) even though hypothyroid patients were less than half as likely to be taking a calcium channel blocker or beta-blocker (31\% vs $75 \%)$.

\section{Discussion}

Our study was prompted by reports that surgical outcomes may be improved with pre-operative assessment of patient comorbidities (see, for example [18]). However, these reports generally do not provide advice on how to handle patients found to be hypothyroid. Currently routine testing for hypothyroidism is not recommended as part of the pre-operative assessment. However, if a new diagnosis of hypothyroidism is discovered, delay of surgery has been recommended in patients with "overt symptoms, significant clinical findings of hypothyroidism, or very low thyroid hormone levels" [19]. However, precise thresholds of what constitutes very low thyroid hormone levels or "significant" clinical symptoms have not been established. Neither has it been established on how to best weigh these risks against the urgency of the surgery under consideration. Thus, the assessing physician is often left in a quandary as to whether to recommend postponement of surgery while waiting for treatment of the hypothyroidism. The majority of the hypothyroid patients in our study had thyroid testing done either on the day of surgery (eight of twenty-nine) or prior to surgery (twelve of twenty-nine). Possibly, because most of the procedures were necessary or urgent in nature, the evaluating physician may have felt the benefit of proceeding with surgery outweighed the risk. Several patients (nine of twenty-nine) had their thyroid function tests drawn within a few days after surgery (all but one within one to five days of surgery with the longest interval in one patient of ten days post) and so were not evaluated preoperatively. We speculate that because several of the patients had a known history of hypothyroidism and had been prescribed thyroid medication, the attending physician assumed these patients were adequately replaced. Nevertheless, presuming this assumption were correct, we felt it was important to entertain the possibility that post-op changes in nutrition, medications, or management, may have altered the thyroid function test results. Therefore, a subanalysis was conducted excluding the 9 subjects who had only post-op thyroid function tests. However, similar primary outcome findings persisted with the average length of stay of the remaining 20 hypothyroid patients being 14.0 days compared to the predicted value of 6.9 days. 
Earlier literature does provide evidence of increased risk when operating on patients with significant hypothyroidism [20]. Moreover, there is the suggestion that hypothyroid subjects operated upon for spine surgery may have a 1-day increase in length of stay that was even much longer if the patient had poorly controlled diabetes mellitus [21]. This finding stands in contrast with that of Syed and co-workers who found that there was no significant outcome difference in patients with elevated TSH on thyroid supplement who underwent coronary bypass surgery [22] and to Sherman et al. who found no effect on outcomes in hypothyroid patients undergoing angioplasty [23]. More recent data add further confusion to the outcome of surgery on hypothyroid patients.

One can speculate that the increased length of stay may be related to the severity of hypothyroidism. However, it is important to emphasize the prolonged length of stay of our patients was not correlated with the TSH value. Thus, the increased morbidity noted in this study is not related to the degree of hypothyroidism, in as much as the TSH is an indicator of the degree of hypothyroidism. Rather, it suggests it is related to the presence versus absence of hypothyroidism per se. This observation suggests that consideration of postponing surgery to correct hypothyroidism should be given to all hypothyroid patients. Komatsu et al. found no association of hypothyroidism with length of stay or other complications in patients undergoing cardiac surgery [24]. However, other papers have reported significant increase in complications for valve replacement [25] or higher mortality in hypothyroid subjects with soft-tissue infections [26].

We recognize that there are several potential weaknesses with our study. The study was retrospective in nature and the number of subjects we studied was relatively small. However, there are a number of strengths of our study as well. We carefully reviewed the charts of all the study and control subjects to assure the data and conclusions were accurate. We also did not limit the type of surgery to one specialty or even to one hospital within our network. Thus, our findings are generalizable. Our observations indicate that, in contrast to our initial hypothesis, hypothyroidism is associated with markedly increased length of hospital stay. However, despite a trend toward increased risk of several secondary outcomes, the absolute incidence of these did not significantly differ. This study was not sufficiently powered to discern differences in mortality or post-operative cardiac complications. It does indicate however that, even with modern techniques and anesthesia, there remains a significantly increased risk of operating on a hypothyroid patient. We believe this would be manifested by increased burden on the patient both monetarily and through consequences to their health as well as in the cost to the institution in facility fees and manpower. While hypothyroidism may not be an absolute contraindication to necessary procedures, we suggest that length of stay in such patients may be prolonged by $50 \%$. Therefore, elective surgeries should be delayed until the patient is euthyroid. Whether pre-operative assessment should include a measurement of TSH will require a larger study to assess the overall risks and benefits to hypothyroid patients. It would also be 
valuable to compare hypothyroid patients whose surgery was postponed to achieve euthyroid status with those whose surgery was not postponed.

\section{Acknowledgements}

This paper was presented at the 87th Annual Meeting of the American Thyroid Association, October 18-22, 2017. We gratefully appreciate the help of Elaine Bammerlin in preparation of the Tables and Figures and editorial help.

\section{Conflicts of Interest}

The authors have no conflict of interests to declare.

\section{References}

[1] Wijeysundera, D.N., Austin, P.C., Beattie, W.S., Hux, J.E. and Laupacis, A. (2010) Outcomes and Processes of Care Related to Preoperative Medical Consultation. Archives of Internal Medicine, 170, 1365-1374.

https://doi.org/10.1001/archinternmed.2010.204

[2] Clelland, C., Worland, R.L., Jessup, D.E. and East, D. (1996) Preoperative Medical Evaluation in Patients Having Joint Replacement Surgery: Added Benefits. The Southern Medical Journal, 89, 958-960. https://doi.org/10.1097/00007611-199610000-00004

[3] McGinn, T., Conte, J.G., Jarrett, M.P. and ElSayegh, D. (2005) Decreasing Mortality for Patients Undergoing Hip Fracture Repair Surgery. The Joint Commission Journal on Quality and Patient Safety, 31, 304-307. https://doi.org/10.1016/S1553-7250(05)31038-5

[4] Elixhauser, A., Steiner, C., Harris, D.R. and Coffey, R.M. (1998) Comorbidity Measures for Use with Administrative Data. Medical Care, 36, 8-27. https://doi.org/10.1097/00005650-199801000-00004

[5] Maldonado, L.S., Murata, G.H., Hershman, J.M. and Braunstein, G.D. (1992) Do Thyroid Function Tests Independently Predict Survival in the Critically Ill? Thyroid, 2, 119-123. https://doi.org/10.1089/thy.1992.2.119

[6] Mingote, E., Merono, T., Rujelman, R., et al. (2012) High TSH and Low T4 as Prognostic Markers in Older Patients. Hormones (Athens), 11, 350-355. https://doi.org/10.14310/horm.2002.1364

[7] Lanaro, E., Caixeta, A., Soares, J.A., et al. (2014) Influence of Gender on the Risk of Death and Adverse Events in Patients with Acute Myocardial Infarction Undergoing Pharmacoinvasive Strategy. Journal of Thrombosis and Thrombolysis, 38, 510-516. https://doi.org/10.1007/s11239-014-1072-7

[8] Stathatos, N. and Wartofsky, L. (2003) Perioperative Management of Patients with Hypothyroidism. Endocrinology \& Metabolism Clinics of North America, 32, 503-518. https://doi.org/10.1016/S0889-8529(03)00007-0

[9] Weinberg, A.D., Brennan, M.D., Gorman, C.A., Marsh, H.M. and O’Fallon, W.M. (1983) Outcome of Anesthesia and Surgery in Hypothyroid Patients. Archives of Internal Medicine, 143, 893-897. https://doi.org/10.1001/archinte.1983.00350050047010

[10] Ladenson, P.W., Levin, A.A., Ridgway, E.C. and Daniels, G.H. (1984) Complications of Surgery in Hypothyroid Patients. The American Journal of Medicine, 77, 261-266. https://doi.org/10.1016/0002-9343(84)90701-0 
[11] Wartofsky, L. (2006) Myxedema Coma. Endocrinology \& Metabolism Clinics of North America, 35, 687-698. https://doi.org/10.1016/j.ecl.2006.09.003

[12] Appoo, J.J. and Morin, J.F. (1997) Severe Cerebral and Cardiac Dysfunction Associated with Thyroid Decompensation after Cardiac Operations. The Journal of Thoracic and Cardiovascular Surgery, 114, 496. https://doi.org/10.1016/S0022-5223(97)70201-0

[13] Bilimoria, K.Y., Liu, Y., Paruch, J.L., et al. (2013) Development and Evaluation of the Universal ACS NSQIP Surgical Risk Calculator: A Decision Aid and Informed Consent Tool for Patients and Surgeons. Journal of the American College of Surgeons, 217, 833-842. https://doi.org/10.1016/j.jamcollsurg.2013.07.385

[14] Clark, D.E., Fitzgerald, T.L. and Dibbins, A.W. (2018) Procedure-Based Postoperative Risk Prediction Using NSQIP Data. Journal of Surgical Research, 221, 322-327. https://doi.org/10.1016/j.jss.2017.09.003

[15] Sellers, M.M., Merkow, R.P., Halverson, A., et al. (2013) Validation of New Readmission Data in the American College of Surgeons National Surgical Quality Improvement Program. Journal of the American College of Surgeons, 216, 420-427. https://doi.org/10.1016/j.jamcollsurg.2012.11.013

[16] Rivard, C., Nahum, R., Slagle, E., Duininck, M., Isaksson Vogel, R. and Teoh, D. (2016) Evaluation of the Performance of the ACS NSQIP Surgical Risk Calculator in Gynecologic Oncology Patients Undergoing Laparotomy. Gynecologic Oncology, 141, 281-286. https://doi.org/10.1016/j.ygyno.2016.02.015

[17] Team, R.C. (2018) R: A Language and Environment for Statistical Computing. R Foundation for Statistical Computing, Vienna.

[18] Halaszynski, T.M., Juda, R. and Silverman, D.G. (2004) Optimizing Postoperative Outcomes with Efficient Preoperative Assessment and Management. Critical Care Medicine, 32, S76-S86. https://doi.org/10.1097/01.CCM.0000122046.30687.5C

[19] Jaffer, A. and Grant, P. (2012) Perioperative Medicine, Medical Consultation and Co-Management. Wiley-Blackwell, Hoboken. https://doi.org/10.1002/9781118375372

[20] Worku, B., Tortolani, A.J., Gulkarov, I., Isom, O.W. and Klein, I. (2015) Preoperative Hypothyroidism Is a Risk Factor for Postoperative Atrial Fibrillation in Cardiac Surgical Patients. Journal of Cardiac Surgery, 30, 307-312.

https://doi.org/10.1111/jocs.12513

[21] Walid, M.S. and Zaytseva, N. (2010) How Does Chronic Endocrine Disease Affect Cost in Spine Surgery? World Neurosurgery, 73, 578-581. https://doi.org/10.1016/j.wneu.2010.02.066

[22] Syed, A.U., El Watidy, A.F., Akhlaque, N.B., et al. (2002) Coronary Bypass Surgery in Patients on Thyroxin Replacement Therapy. Asian Cardiovascular and Thoracic Annals, 10, 107-110. https://doi.org/10.1177/021849230201000203

[23] Sherman, S.I. and Ladenson, P.W. (1991) Percutaneous Transluminal Coronary Angioplasty in Hypothyroidism. The American Journal of Medicine, 90, 367-370. https://doi.org/10.1016/0002-9343(91)90578-L

[24] Komatsu, R., Karimi, N., Zimmerman, N.M., et al. (2018) Biochemically Diagnosed Hypothyroidism and Postoperative Complications after Cardiac Surgery: A Retrospective Cohort Analysis. Journal of Anesthesia, 32, 663-672. https://doi.org/10.1007/s00540-018-2533-5

[25] Subahi, A., Yassin, A.S., Adegbala, O., et al. (2018) Comparison of Hospital Outcomes of Transcatheter Aortic Valve Implantation with versus without Hypothyroidism. American Journal of Cardiology, 122, 838-843. 
https://doi.org/10.1016/j.amjcard.2018.05.025

[26] Latifi, R., Patel, A.S., Samson, D.J., et al. (2019) The Roles of Early Surgery and Comorbid Conditions on Outcomes of Severe Necrotizing Soft-Tissue Infections. European Journal of Trauma and Emergency Surgery, 45, 919-926.

https://doi.org/10.1007/s00068-018-0958-Z 